\title{
Retention of protoplanetary cores near the snowline
}

\author{
Xiaojia Zhang ${ }^{1}$, Katherine Kretke ${ }^{3}$ and D. N. C. Lin $^{2,3}$ \\ ${ }^{1}$ Department of Astronomy, PKU, China, \\ ${ }^{2}$ KIAA, PKU, China, \\ ${ }^{3}$ Department of Astronomy and Astrophysics, UCSC, CA
}

\begin{abstract}
Suppression of type I migration is essential for the retention of protoplanetary cores which are sufficiently massive to accrete gas in their nascent disks and evolve into gas giant planets. We explore here the possibility that special disk properties at the snow line may be the dominant process which stalled the type I migration. We simply use a 1-D model to calculate the torque with linear formula and find that, if the surface density jump near snowline is great enough, the migration can be efficiently slowed down or even halted. This mechanism offers an explanation to the observed peak, at 2-3 AU, in the extra solar planets' semi major axis distribution.
\end{abstract}

Keywords. Protoplanetary cores, Type I migration, Snowline

\section{Introduction}

Existence of hot Jupiters tell us that planetary migration due to the interaction between disk and planet is important for planetary formation (Lin et al. 1996). For a low mass planet which can not strongly perturb the disk density profile, the linear density waves excited by it will exerts torques on the planet(Goldreich \& Tremaine 1979). The total torque is negative causing an inward orbital migration of the planet, which is called Type I migration(Ward 1997). According to the result of Tanaka et al. (2002), consider an Earth-mass planet around a sun-mass object and a low-mass protoplanetary disk model, the Type I migration time is about $10^{5}-10^{6} \mathrm{yr}$, which is shorter than the typical lifetime of the disk. Since terrestrial planets could form after gas was depleted, the type I migration is not a problem for them. However, it is a challenge for the gas giants because their formation must be preceded by the emergence of several Earth mass cores (Pollack et al. 1996) in a gaseous medium. With type I migration, these cores would not survive. But, the observation results challenge the planetary migration theory (Figure 1). There is such a significant fraction of solar type stars have gas giants and there is an enhancement in the period distribution around Snowline at 2-3 AU.

\section{Method}

The special profile near the Snowline (Figure 2) is due to the suddenly viscosity change. Given that the turbulence viscosity comes from MHI (Magneto-Rotation Instability), The depth to which MRI effectively generates turbulence is a strong function of grain size and abundance. A sharp increase in the grain-to-gas density ratio across the snow line reduces the depth of the active layer, and then reduce the viscosity sharply resulting in a local maximum value of gas surface density (Kretke \& Lin 2007). Across the snow line, the change in the latent heat reduces the local temperature gradient such that the local surface density gradient also corresponds to a local pressure maximum. We use 
a 1-D isothermal disk model with the surface density profile showed in Figure 2, and the temperature $T=166 \mathrm{~K}$. In a centrifugal balance, gas flow is super Keplerian in regions with a positive radial pressure gradient (slightly interior to the snow line) and sub Keplerian in regions with a negative radial pressure gradient (all other locations in the disk).

We consider an Earth-mass core around a sun-mass object and take protoplanetary core's orbit as a circle. Since the core has not sufficient mass to open a gap, we neglect the core's disturbance on the disk structure and use alpha expression for the viscosity as $\nu=\alpha c_{s} H$, where $c_{s}$ is sound speed and $\mathrm{H}$ is the height of disk. The total torque exerted on a planetary core is the sum of the torques arising from the inner and outer Lindblad resonances and the corotation resonance:

$$
\Gamma_{t o t}=\sum_{m} T_{m}+\Gamma_{C}
$$

where

$$
T_{m}=-\pi^{2} m \Sigma \frac{\Psi_{m}^{2}}{R\left(\frac{d D_{*}}{d R}\right)}
$$

is the torque from an m-order Lindblad resonance, which is calculated by Ward (1997). $\Sigma$ is the surface density of disk at $\mathrm{R}$ and $\Psi_{m}$ is the forcing function(Goldreich \& Tremaine 1979). $D_{*}=0$ gives the location of an m-order Lindblad resonance. We sum the torques to $m=40$ since the torque arising form very near the protoplanetary core has some uncertainty. And

$$
\Gamma_{C} \propto \Sigma \frac{d \log (\Sigma / B)}{d \log R} \sim h^{-2}\left(\frac{M_{P}}{M_{*}}\right)^{2} R^{4} \Omega_{P}^{2} \Sigma \frac{d \log (\Sigma / B)}{d \log R}
$$

is the corotation torque calculated by Masset et al. (2006). Where $h=H / R, M_{p}$ and $M_{*}$ are the mass of the core and center object respectively, $\Omega_{P}$ is the angular velocity of the core. For a disk with surface density proportional to $r^{p}$ where $p=-1.5, \Gamma_{C}$ is almost zero, but in the situation near the snowline it is significantly non zero. With the total torque one can calculate the Type I migration rate as

$$
\tau_{\text {mig }}=\frac{M_{P} R^{2} \Omega_{P}}{2 \Gamma_{t o t}}
$$

Set the initial location of the core outside the snowline, we can find that the core is

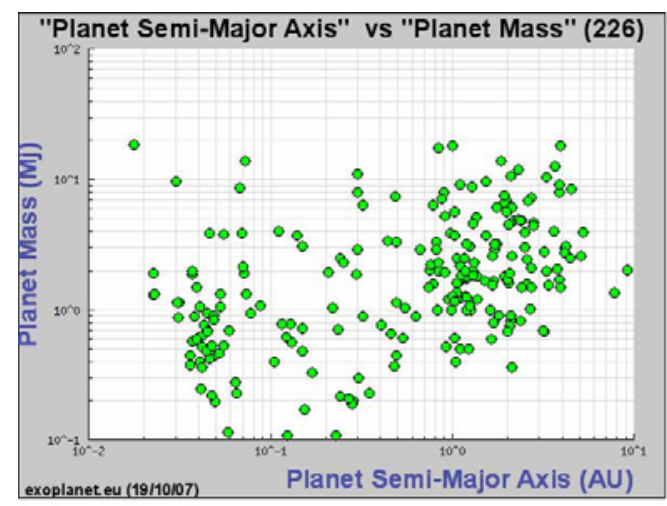

Figure 1. Mass-Semimajor axis distribution of observed exoplanets(exoplanet.edu) 


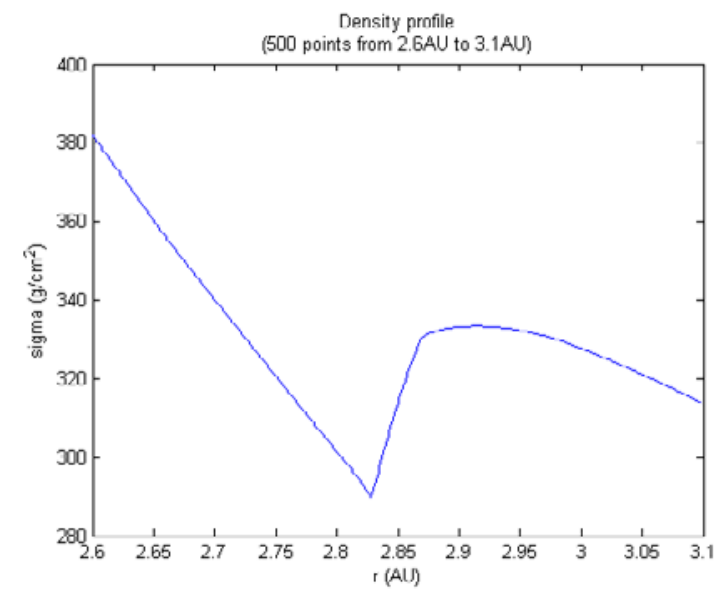

Figure 2. Density profile near snowline generated by Kretke

trapped at the snowline and will not migrate through it (Figure 3). The oscillation is because that the total torque exerted on the protoplanet reverses from negative to positive when get near to the snow line. Therefore the protoplanets will migrate outward and when they get a little further the total torque again becomes negative. So we can see the protoplanet's orbital radius oscillates around the outer edge of snowline. Tanaka et al. (2002) has already given the migration rate as a function of the radial gradient of the disk gas surface density But the expression of Type I migration in the papers of Tanaka et al. (2002) is available for the density profile with a certain gradient. When near the snow line, for a fixed location of protoplanets, different orders of Lindblad torque correspond to different density gradient. We want to accurately calculate the total torque although we can get an estimation from the result of Tanaka et al. (2002). We also use this method to calculate the migration rate for a normal disk model, by comparing the result with that of Tanaka et al. (2002) we find the method is reasonable.

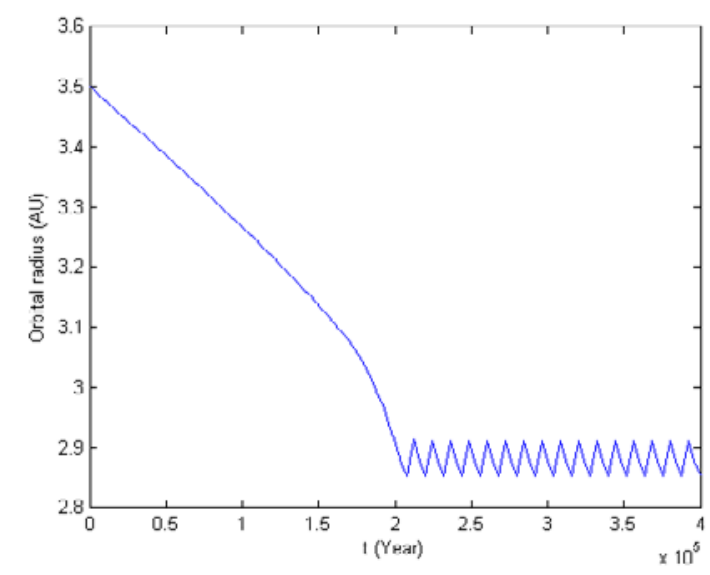

Figure 3. Orbital evolution due to Type I migration of an Earth-mass protoplanetary core. 


\section{Summary}

Protoplanetary cores would migrate inward with a normal disk profile, but when they get close to Snowline, the torque exerted on them may change from negative to positive. Then the cores will be trapped around the Snowline. Comparing with observation result of exoplanets' semi major axis distribution, we can roughly find two groups: Protoplanetary cores formed and retained near the snowline can be explain by this result. And the smaller group may have undergone orbital decay due to Type II migration(Ida \& Lin 2004).

\section{References}

Goldreich, P. \& Tremaine, S. 1979, ApJ, 233, 857

Ida, S. \& Lin, D. N. C. 2004, ApJ, 604, 3881

Ida, S. \& Lin, D. N. C. 2004, $A p J, 616,5671$

Kretke, K. A. \& Lin, D. N. C. 2007, ApJ, 664L, 55K

Lin, D. N. C., Bodenheimer, P., \& Richardson, D. C. 1996, Natur, 380, 606L

Masset, F., Morbidelli, A., Crida, A., \& Ferreira, J. 2006, ApJ, 642, 478M

Pollack, James B., Hubickyj, Olenka, Bodenheimer, Peter, Lissauer, Jack J., Podolak, Morris, \& Greenzweig, Yuval 1996, Icar, 124. 62P

Tanaka, H., Takeuchi, T., \& Ward, W. R. 2002, ApJ, 565, 1257

Ward, W. R. 1997, Icar, 126, 261W 